\title{
MOBILE ADHOC NETWORK LINKAGE ACCESSIBILITY \& POWER CONTROL CROSS LAYER DESIGN
}

\author{
Md. Sajid Anwer ${ }^{1} \&$ Syed H Hasan ${ }^{2}$
}

\begin{abstract}
Mantes face the ever-present key challenge of battery power that is limited along with mobility caused network topology changes that are frequent. Power depletion in network nodes may be the root of nodes going offline, thus affecting the links inside the network. Node mobility also presents the issue of frequent breakage of established routes, in-turn affecting the performance of the applications dependent on the network. In this research we are suggesting as a solution for the issues of link availability and power conservation combined. Hence we combine the effects of AODV protocol with link availability based on optimum received and transmit signal strength/power through the use of cross-layered-approach. In this research we propose link prediction based on received signal strengths to improve link-availability and using optimal power to transmit packet to a node in the neighborhood for increasing the adhoc node battery life. We propose strength of received signals and transmit power as parameters based on cross layer interaction so that link availability is increased through reliable routing and conserved power. This brings an additional increment in the capacity and lifetime of the node $\&$ network. The model also improves ratio of packet delivery and increased network throughput through spatial reusing instant route repairing and predicting breakage of links in advance. Reduction of power consumption along with end-to-end delays is achieved by optimal transmit power usage. Simulations would exhibit better performance achieved through the model suggested.

Keywords - DPCPLP , mobility, link prediction, medium access control, signal strength, power control, routing, MANET, Cross layer.
\end{abstract}

\section{INTRODUCTION}

Mobile devices that communicate in absence of centralized infrastructure and self organize are called MANETs. MANET has nodes acting as packet forwarders to next hop in a multi hop environment as well as routers for delivering the packets to their destinations.

Mobility necessitates, untethered wireless connectivity between each node present in the MANET. Operations of the mobile nodes are dependent on the battery power, hence we need to have minimal power usage by these nodes. The issue gets aggravated, since post battery exhaustion the node can neither receive or send data. A node dying out would negatively impact network performance, since in MANETs each node is important for maintaining connection \& links. In situations where an intermediary node dies, the entire route needs to be build up again. When one of the nodes in between is dead, the entire link needs to be reconstructed. This process is a waste of battery and causes delays which hampers network throughput. Additionally, mobility requires continuous reconfiguration of topology that must be handled through a routing system that is efficient and complex.

Personal communications devices are the primary users of wireless networks, since they can be carried around easily. This would require new applications to be developed. In order to run these applications that are constrained by resources, efficient networking stacks are required on these mobile devices. We have seen use of layered architecture for simplifying the complex job of network connectivity handling. The concept of software components in layers is being introduced to improve performance further, which means non-immediate layers can access data structure in other layers. This methodology is also known as Optimization through cross layers.

MANET QoS would essentially mean assured packet delivery in correspondence with specific flow at higher prioritization in order to satisfy delay and loss performance requirement. The nodes in MANET operate on residual battery charge, who's availability varies widely from node to node. The nodes may be on the move causing link breakage caused by low battery or mobility of nodes on optimal path. Hence for guaranteed QoS across unreliable link, proactive or fast routing recovery, with optimization of application and transport layer is required. In order to avoid power wastage by transmitting of packets over failed links measurements are required at MAC and Data link layer.

In Section 2 of this paper we will discuss similar work done. Next section 3 would suggest the methodology of power control for power consumption reduction and estimation of link availabilities. Analysis and simulation results are provided in Section 4 while in Section 5 we have provided the summary.

\footnotetext{
${ }^{1}$ Department of Computer Science, AISECT University, Bhopal, India

${ }^{2}$ King Abdulaziz University, Jeddah, Saudi Arabia.
} 


\section{SIMILAR WORK}

Problems like reduced network throughput, high power consumption, exposed node and hidden node have been tackled by various researchers by different cross layered mechanism at the Routing and MAC layer levels. We also see use of link prediction at level of network layer. Energy conservation has been sought to achieve through combining optimizations at multiple layers. We would discuss some of the cross layered, routing and MAC optimization mechanism.

\subsection{Cross Layered Protocols}

Wireless MANETs cannot be optimized efficiently at different layers because of channel conditions that are unpredictable and resources that are limited and dynamic. Hence cross layer optimization through use of information available across layers is a must for MANETs for obtaining optimum results. Here information exchange among layers happens for improving the overall performance of the network.

Interaction between Network and Physical layer: [6] studies the 5 routing protocols being impacted by the physical layer. Network performance is much better when properties of physical layer like shadowing and path loss are considered instead of traditional scenarios. The study established that just the hop-count solely should not be considered for routing, instead MANETs routing should also be based on link quality and current channel state.

Interaction between Transport and Physical layer: Mobile node transmission rate are often influenced by power control. [7] explores the balancing of transport layer congestion control and physical layer power control for enhancing communication over multiple hops. JOCP an algorithm for distributive power controlling is coupled by traditional TCP for bringing improvement in energy efficiency network throughput. JOPC essentially proposed updating of transmission power by nodes at bottleneck links for faster packet transmission during periods of congestion.

Interaction between MAC and Physical layer: TCP's poor performance in MANETs is mainly caused by it's inability of distinguishing congestion packet loss and link fluctuation, mobility etc. packet loss. Some researchers proposed to modify the TCP and MAC separately to solve this problem others have suggested tackling the issue at both the fronts combined. [8] presents problem of degradation of performance at transport layer caused by congestion. C3TCP (scheme of congestion control across layers) gave better performance through considering data such as link layer delay and bandwidth capacity.

We find combined optimization of scheduling, routing and congestion control across layers in MANETs presented at [9]. Scheduling constraints and rate constraints are used on the basis of flow variables and are formulated upon resource allocations in network that have wireless channels fixed. Problem of resource allocation has been sub-divided into : Scheduling, routing and congestion control.

\subsection{Protocols for Routing}

MANET's Routing protocols usually consists of route maintenance and discovery mechanisms. The discovery system is used for identifying a possible route between the receiver and the sender while the maintenance part of the protocol identifies new routes in case the existing routes are broken due to un-controllable reasons.

One of the on demand protocols for routing is AODV [3]. Whenever, a node intends to transmit a message to specific node, and route is not available to the destination, a path discovery is initiated by the sender through RREQ(Route REQuest) packet. A recipient of RREQ determines if the packet has been received in the past, if yes, the packet is discarded. If the packet is new it check for a pre-existing route to destination in cache. If the RREQ cannot be satisfied, the packet is rebroadcasted with reverse path information to the source. Reverse path is determined by including the address of the node from which the RREQ was received. Every intermediary node passes the packet down the chain until it reaches the destination node. The destination unicasts the RREP(Route REPly) packet to the original sender node. Every node that passes the RREP packet upstream includes the information of the node from which the RREP packet is received as a forward pointer along with timeout informations. Thus the entire chain as the latest updated information from source to destination in its cache. Nodes that do not fall on the RREP path delete the information after the "ACTIVE_ROUTE_TIMEOUT".

When a unreachable destination node is encountered by a sender node (non-reception of Hello message/acknowledgement from link layer), a RERR(Route ERRor) packet is sent to every active neighboring node that fell on the path to the destination node.

Active neighbor list is maintained for every route in the cache. Active neighbors the nodes that have send a minimum of 1 packet to the destination node within last ACTIVE_TIMEOUT duration. Every route in the cache is awarded a destination sequence number to prevent routing loops, even in rare conditions like high mobility of nodes or packet deliveries that are out of order. Sequence number also facilitates to check route freshness, with the most recently updated routes having the highest sequence number.

Packet delivery is negatively impacted significantly by route failures. High delays and dropping of packets are mainly resulted because of route failure. Traditional routing normally have high gaps between the detection of broken links and establishing of alternative routes. Hence flow downtime reduction is important.

The preemptive routing proposed In [4], Goff proposed preemptive routing that can estimate the link breakage based on strength of received signals. A route discovery is initiated and fresh link established prior to link failures. $\delta$ is the preemptive ration that is utilized to define the zone of preemption with respect to the threshold of signal strength. Geoff applied this mechanism of preemptive route discovery and maintenance AODV and DSR. Wireless networks normally adjust the power 
of transmission to ensure delivery of signals to receiving nodes. However, this adjustment of transmission power was not part of his work.

A number of mechanisms were proposed for identifying stable and long lasting links thus reducing resource consumptions. In [5] LAER proposed route discovery to use a joint metric for rate of energy drain and link stability. This led to reduction in control overhead and balancing of traffic loads.

\subsection{Protocol at MAC layer}

The standard protocol for wireless network ad defined by IEEE $802.11 \mathrm{~b}$ is defined in [1] as DCF MAC Protocols. Working of the protocol is shown in Fig 1. This protocol uses virtual sensing concept through a 4-way handshake. Communication duration is contained in CTS and RTS. When a non-destination node receives a packet then received value is set as it's Network Allocation Vector(NAV) and transmission deferred for the same time duration. Collision packets are reduced and channels reserved through the virtual sensing mechanisms. Nodes that are unsucessfull transmit again once the backoff period is over. All communications are done at maximum power levels, hence draining the battery significantly. When a CTS or RTS packet is not received properly by a node collisions still happen because the node's NAV field is not updated correctly.

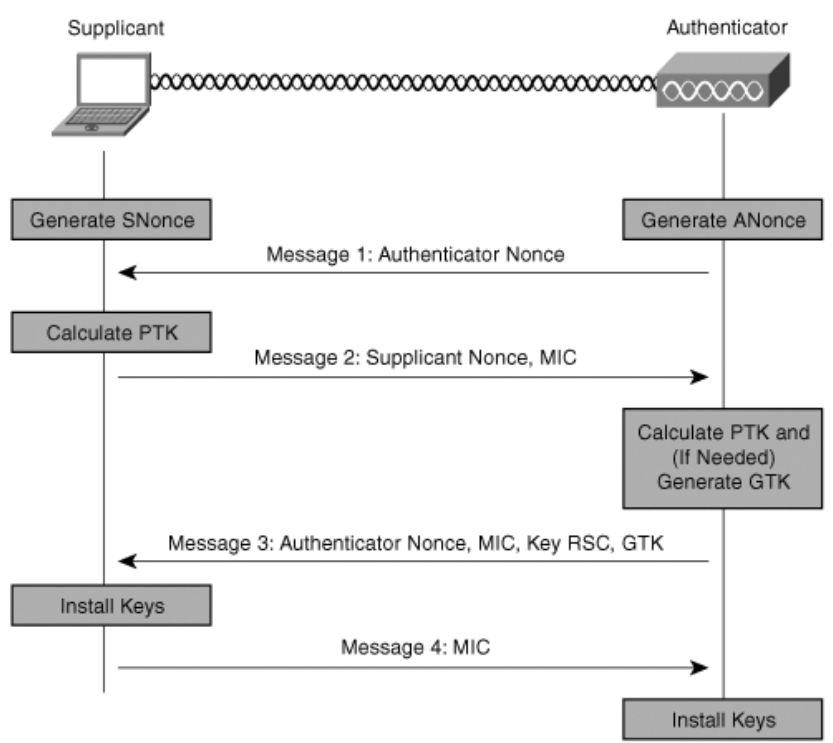

Fig 1: Four way handshakes (RTS-CTS-DATA-ACK) MAC Protocol

In [2] a MAC layer protocol controlled by Adaptive Power proposes methodology for increasing overall network throughput and reducing consumption of power. This protocol transmits packets at just enough power so that they may reach the destined nodes. Received signals are used for estimation of transmission power calculated through:

$P_{\mathrm{t}}=\frac{P_{\min } * P_{\mathrm{t}}}{P_{r}^{\dagger}}$.

Here $\quad P_{t}^{\prime}$ and $P_{r}^{\prime}$ are powers of transmission and reception observed in the communication for the packets earlier. Loss is assumed to be the same in the reverse direction also. Optimum level of power is used for sending all ACK, DATA, CTS, RTS packets.

\section{PREDICTION OF LINK AVAILABILITY AND POWER CONTROL ACROSS LAYERS}

Interaction between network and physical layers (Cross layer) interaction is seen in Fig. 2. Network layer uses the strength of received signal for initiating the process for new route finding.

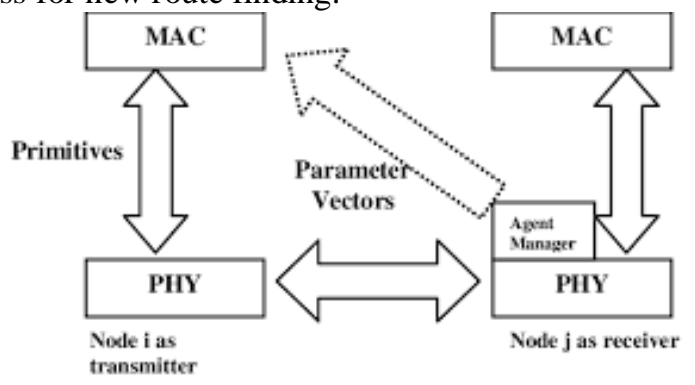

Figure 2: Cross layer interactions at node 
Network capacity and lifetime is increased by DPCPLP(Prediction of Link Availability by Cross layer approach). This is achieved by new path formation before path breakage to support QoS requirements presented by applications, based on estimated link availability time and transmission of ACK, DATA, CTS, RTS packets at optimum levels.

Power control: MAC layer transmits ACK, DATA, CTS, RTS packets at optimum levels enough for sustaining communications at good quality. Transmission power is dynamically adjusted based on historical communication and signal strengths between sender and receiving nodes.

Link availability: Strength of received signals from physical layers is used for estimating the time of link availability and link break predictions are made accordingly. Sources and upstream nodes are pre-warned of link breakage hence enabling them to identify alternative paths beforehand.

\subsection{Power control}

DPCP (Dynamic Power Control Protocol) @ MAC layer is proposed for maximizing mobile node battery life. The proposed protocol has it's basis on MAC Protocol for Adaptive Power Control in a manner that overall power of transmission is low thus reducing cattery consumption.

ACK, DATA, CTS, RTS packets are sent using optimum power. Level of transmission power are contained in the header field of ACK, DATA, CTS, RTS packets that can be utilized for computing the power level that is optimum for sending these packets.

The IEEE 802.11std is reliable MAC protocol. When a sending node transmits RTS, CTS, DATA and ACK packets, every exposed node receives the packet at received signal strength. The received signal strength, $\boldsymbol{P}_{r}$ at receiver using two ray propagation model is:

$$
P_{r}=P_{t} G_{t} G_{r}\left(\frac{\lambda}{4 \pi d_{i j}}\right)^{2}
$$

Where $\lambda$ is the wavelength of carrier, $d_{i j}$ is the distance between sender and receiver. $G_{t}$ and $G_{r}$ are the gain of transmitting and receiving omni directional antennas respectively. The power $P_{t}$ is the transmit power of the packet. The header fields of the packets RTS, CTS, DATA and ACK are modified to incorporate the transmission power level of the respective packets.

Thus when a node receives such packet, it gets the transmission power level $P_{t}$, the received power $P_{r}$ is calculated by the physical layer and the value is send to MAC layer. Every node knows the minimum threshold power $P_{\text {threshold }}$ at which the packet can be decoded properly. Thus we get the desired minimum transmission power required so that packet is properly decoded at the receiver.

$$
P_{\text {tmin }}=\frac{P_{\text {threshold }}}{G_{t} G_{r}}\left(\frac{4 \pi d_{i j}}{\lambda}\right)^{2} \& c
$$

However, we do not have information about distance [2] between two nodes. We can find out optimum transmission power by the equation

$$
\begin{gathered}
P_{\text {tmin }}=\frac{P_{\text {threshold }} * P_{t} * C}{P_{r}} . \\
P_{\text {npt }} \geq P_{\text {tmin }} .
\end{gathered}
$$

Where, $P_{\text {opt }}$ is the optimum transmission power and is discrete level greater than $P_{\text {tmin }}, P_{t}^{\prime}$ and $P_{r}^{\prime}$ are the transmission and received powers of the previous packet from that receiver to sender, respectively. $\mathrm{C}$ is a constant equal to 1.05 to compensate for the interference and noise.

A table would be maintained by every node which contains levels of optimum power of transmission so that destination nodes are able to decode the messages and able to initiate link success processes. Tables would contain two fields MAC Address of destined node and corresponding power levels. We will call is 'Table for Optimum Power' and it would be a small table since it would only contain information about neighboring nodes only 
Here in the design of cross layer, receivers limit level of optimum transmission power that are just enough for sustaining communications of good quality and initiate the link breakage prediction process. 3 thresholds of received signal strength are used by us, namely

threshold received signal strengths $P_{\text {threshold }} P_{\text {critical }}$ and $P_{\text {decode }}$ respectively. At $P_{\text {threshold }}$, the node enters into link prediction process. At $\Gamma_{\text {critical }}$, the node enters into critical state, warns the upstream node about link break and forms alternate path prior to link break. The $P_{\text {desode }}$ is minimum power allowed for the destination node to decode the packet.

Physical layer then receives the information about signal strength and calculates it before passing it to MAC layer for transmission of data packets. Equation (4) is used for computing optimum transmission power. Every nodes' table of optimum power then stores this $P_{\text {opt }}$ against the destination node. Each packet's level of transmission is put into its header through modification of the ACK, DATA, CTS, RTS packet headers for getting the optimum power of transmission in the cross layered design. Hence when such packets are received by any node $P_{t}$, the level of transmission power is received alongwith and $P_{r}$ the received power is obtained from physical layer, the MAC layer is then passed the transmit power that is calculated. The interaction between the MAC and physical layers is clearly indicated here.

Transmit power is inserted as an additional field into RTS by the sending node so that the CTS packet sending power is tuned by the receiving node. The DATA packet from the sending node and the ACK packets from the receiving nodes are also transmitted at optimum power subsequently.

\subsection{Availability of Link}

Strength of received signals from physical layers is used for estimating the time of link availability and link break predictions are made accordingly. Sources and upstream nodes are pre-warned of link breakage hence enabling them to identify alternative paths beforehand. [10].

In this approach, three consecutive measurements of signal strength of packets received from the predecessor node are used to predict the link failure using the Newton divided difference interpolation method. The Newton interpolation polynomial has the following generalized expression.

$$
\left.f(x)=f\left(x_{0}\right)+\left(x-x_{0}\right) f\left(x_{0}, x_{1}\right)+\cdots+c \prod_{i=0}^{n-1}\left(x-x_{i}\right)\right) f\left(x_{0}, x_{1}, \ldots, x_{n}\right) .
$$

The received signal strengths of the three latest data packets and their time of occurrence are maintained by each receiver for each transmitter from which it is receiving. Using three received data packets' signal power strengths as $P_{1}, P_{2}, P_{3}$ and the time when packets arrived as $t_{1}, t_{2}$, $t_{3}$ respectively and $P_{p}$ instants as the decode signal strength $\left(P_{\text {decode }}\right)$ at the time $t_{p}$, one can determine $t_{p}$ using equation (7). We assume that at the predicted time $t_{p}$, when received power level reduces to or less than decode power, the link will break. The expected signal strength of the packets received can be computed as below, where $\Delta$ and $\Delta^{2}$ are first and second divided differences respectively.

$$
\begin{gathered}
P_{p}=P_{1}+\left(t_{p}-t_{1}\right) \Delta+\left(t_{p}-t_{1}\right)\left(t_{p}-t_{2}\right) \Delta^{2} . \\
P_{p}=P_{1}-\frac{\left(t_{y}-t_{2}\right)\left(R_{z}-P_{2}\right)}{\left(t_{2}-t_{\nu}\right)}+\left(t_{p}-t_{1}\right)\left(t_{p}-t_{2}\right)\left(\frac{\left(p_{2}-P_{z}\right)}{\left(t_{2}-t_{2}\right)}-\frac{\left(P_{1}-P_{2}\right)}{\left(t_{2}-t_{2}\right)}\right) /\left(t_{2}-t_{1}\right) .
\end{gathered}
$$

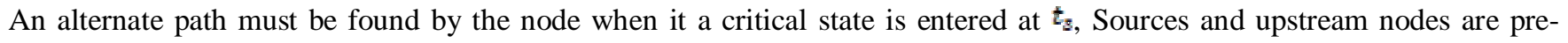
warned of link breakage, where this link is used in the flow. Link discovery mechanisms are invoked by the source nodes for restoration path setups. Received power at $t_{s}$ is the threshold power and is sufficient to send the warning message to the sources and upstream bodes so that they may discover alternative paths and set them up.

\section{ANALYSIS OF SIMULATION RESULTS}

In AODV, AODVLP (AODV with link prediction) and DPCPLP (Dynamic power controlled protocol with link prediction) were simulated with the use of ns-2. Node count, network load and node velocity were varied in the simulation. Table 1 mentions the details of the simulation parameters. We ran number of simulation with below mentioned parameters and then took average of resultant values for reduction of estimation errors.

Model of 2-ray propagation and 7 radio transmit power levels were used. Node density, network load and node velocity were varied, where network load is defined at the rate which packets are generated in the network. 
Table 1 Parameter details

\begin{tabular}{|l|l|}
\hline Traffic Pattern & Constant Bit Rate \\
\hline Velocity & $5,10,15,20,25$ and $30 \mathrm{~m} / \mathrm{s}$ \\
\hline Pause time & 11 seconds \\
\hline Simulation area & $1400 \mathrm{~m}$ by $250 \mathrm{~m}$ \\
\hline Simulation time & 800 seconds \\
\hline Packet size & 512 Bytes \\
\hline Total Connections & $15,20,25,30,35,40$ and 45 \\
\hline Total Nodes & $20,40,60,80$ and 100 \\
\hline
\end{tabular}

Performance measurement is done based on the average throughput, energy consumption, overhead packets, interruption time, packet delivery ratios and end-2-end delay with respect to the mobility of nodes. Sources for CBR are assumed.

Throughput - measured as total kbs of successful data transfer between the sender and receiver.

Energy Consumption/kb data (Joules)- measured a total amount of energy consumed for all data flows divided by total amount of data transmitted for all data flows. Consumption for all kind of packets ACK, DATA, CTS and RTS were taken into account.

Routing overhead - measured as packets generated as routing overhead for transfer of data packet inside the network.

Interruption time - measured as average time for which the communications were interrupted in the network.

Packet delivery ratios - measured as ratio between the packet successfully delivered to destination against number of packets CBR sources generated. A high value indicates better performance here.

End-2-end delay - measured as total delay in the packet delivery that could be because of any contributing factor like transfer and propagation time, MAC layer retransmission delays, interface queue queuing, route discovery bufferings etc.

DPCPLP, AODVLP, and AODV were tested in the simulation. results are obtained for AODV, AODVLP and DPCPLP. We varied the velocity discretely at increments of $5 \mathrm{~m} / \mathrm{s}$ starting with $5 \mathrm{~m} / \mathrm{s}$ and ending with $30 \mathrm{~m} / \mathrm{s}$, 10 second pauses were taken over a fixed network that had 50 nodes, Fig 4 \& 5 display the results. In Fig 3 we compare the Node Velocity with Probability of nodes. Avg. of interruption time between the DPCPLP, AODVLP, and AODV schemes. Avg. interruption time for DPCPLP was least in comparison to AODV and AODVLP. This is primarily due to DPCPLP using transmission range that are smaller which enable concurrent packet transmission, also use of backup paths in DPCPLP enables faster path restoration in cause of link breakage. However we saw an increase in interruption time when the node velocity was increased since faster node mobility would result in more instances of route un-availability. Occurrence of Route unavailability is directly proportional to the interruption time.

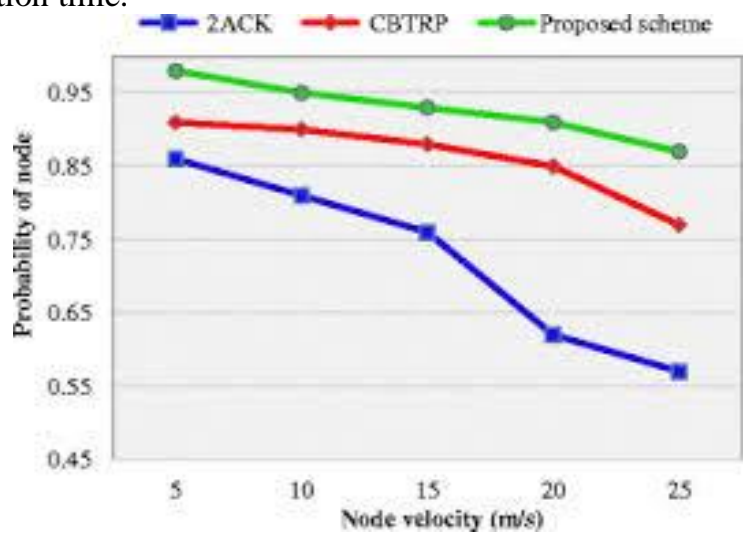

Fig. 3 Node Velocity Vs Probabilty of nodes

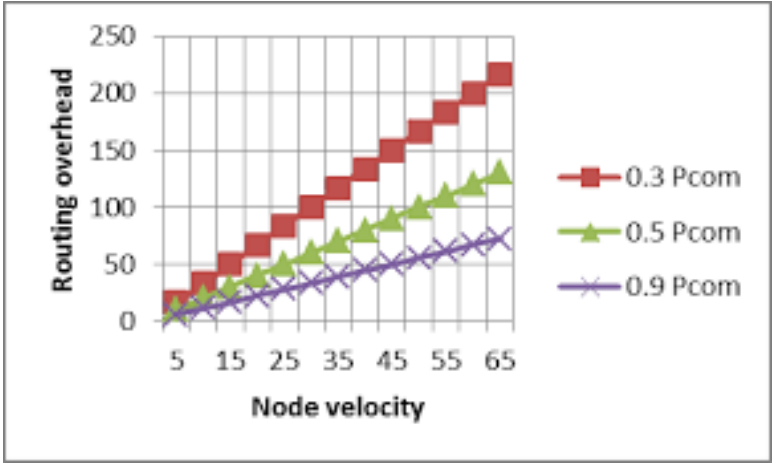

Fig 4 Node Velocity Vs Routing overhead 
In Fig 4 it is evident that DPCPLP has the least number of overhead packets in comparison to AODV and AODVLP. This is achieved by concurrently transmitting packets since the range for carrier sensing is small, additionally route failures occurring because of fast node mobility are covered by available alternate routes. However, similar to the interruption time the high mobility of nodes cause an increase in the number of overhead routing packets in DPCPLP, AODVLP, and AODV schemes. When the node velocity was increased since faster node mobility would result in more instances of route unavailability. Occurrence of Route unavailability is directly proportionate to overhead discovery packets being sent out for finding new routes.

In Fig 5 shows results for varied rate of packet generation with constant velocity of $5 \mathrm{~m} / \mathrm{s}, 10$ second pauses were taken over a fixed network that had 50 nodes. Fig 6 exhibits DPCPLP has least interruption time in comparison to AODV and AODVLP, since it has path availability even in increased packet flows. This is possible since there are alternate paths available in case of link breakage, as well as ACK, DATA, CTS and RTS packets being transmitted with optimum power. However, interruption time increased with increment in the rate of packet generation in all schemes. Since less packets are contending for transmission at lower rates of packet generation but when the generation rates are increased more packets content for being transmitted causing increase in interruption time. This leads to increase in Avg. Interruption time when packet generation rates are increased in DPCPLP, AODVLP, and AODV schemes.

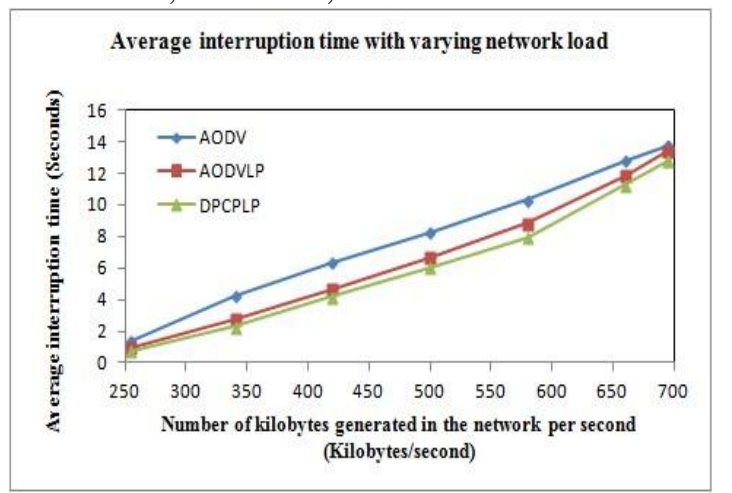

Fig 5 Packet generation rates Vs Avg. interruption time

Fig 6 displays DPCPLP, AODVLP, and AODV schemes throughput compared. Here also we can see higher throughput achieved by DPCPLP when compared to AODVLP, and AODV schemes. shows the comparison of the throughput of AODV, AODVLP and DPCPLP. This is achieved by concurrently transmitting large number of packets since the range for carrier sensing is small. AODVLP also exhibits a higher throughput in comparison to AODV. This is possible since AODVLP and DPCPLP, has additional backup routes discovered prior to link breakage and packets are delivered through these alternative routes when the path is actually broken. Yet, DPCPLP shows increased throughput with increase in the rate of packet generation and the network is more saturated. After a certain rate the throughput becomes almost constant. Since less packets are contending for transmission at lower rates of packet generation but when the generation rates are increased deferring nodes are reduced because of power reduction, hence data transfer per joule is more. This results in linear increase in throughput at higher rates of packet generation which is saturated after certain limits.

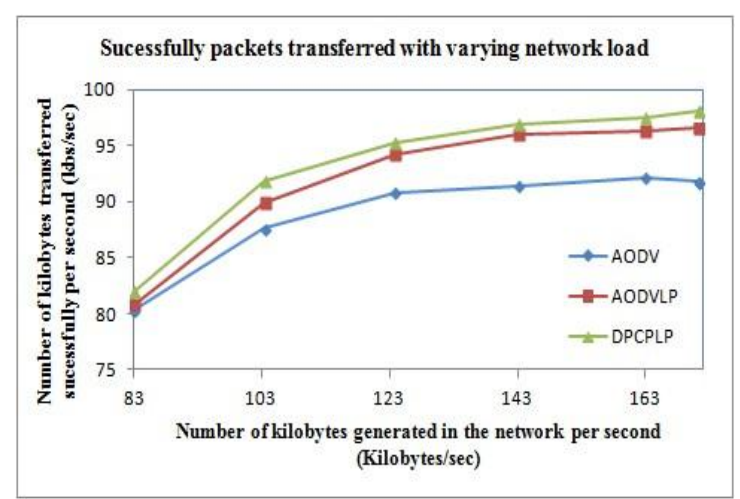

Fig 6 Packet generation rates Vs Throughput 
Fig 7 delimits less energy consumption by DPCPLP for transmitting $1 \mathrm{~Kb}$ of data as it saves energy by using optimum transmission power. Increased node density shows increase in power consumption for all schemes since collision and contention increase with density of nodes in the network. However, consumption of energy at every density of nodes is least in DPCPLP making it most energy efficient protocol.

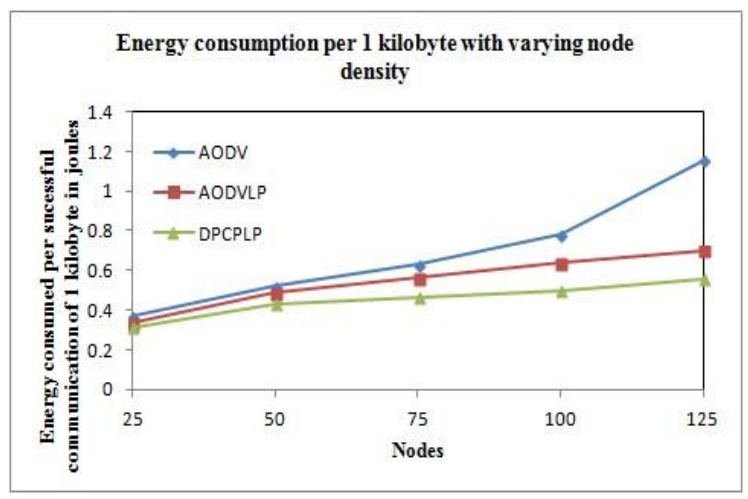

Fig 7 Node Density Vs Avg energy for every $1 \mathrm{~Kb}$ data transfer

Fig 8 shows comparison of ratio of packet delivery and end-2-end delay in the schemes with increase in the node density, respectively. DPCPLP outperforms both the other protocols in each of these categories as well. This is because of concurrent transmission of packets along with availability of alternate routes before path breakage causing more packets to be delivered (Delivery ratio) in less time (end-2-end delay). With increase in the density of the node the delivery ratio falls for all schemes along with increased end-2-end delay. This is caused by the simple fact that since there are more nodes in the network chances of collision and contention increases as well. This causes packets to be retransmitted that results in lower delivery ration and higher end-2-end delays.

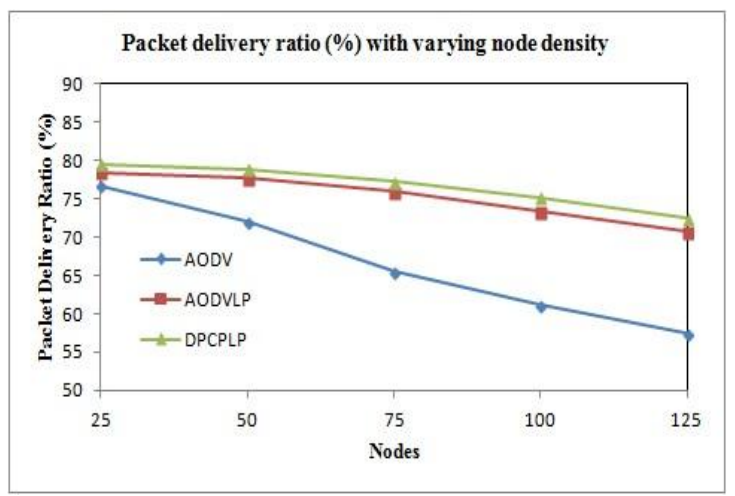

Fig 8 Node Density Vs Delivery ratio

\section{FUTURE WORK AND SUMMARY}

This research has made a proposition of using cross-layered design for providing solutions for power conservation and managing link availability (DPCPLP) in MANETs. This is achieved by adding of MAC layer power control to reduce consumption of power yielding higher battery life along with link breakage prediction function that also repairs/establishes alternate links at the level of network layer. This prediction is based on the signal power threshold and previous 3 consecutive signal strength received MAC layer transmits ACK, DATA, CTS, RTS packets at optimum levels enough for sustaining communications at good quality. Transmission power is dynamically adjusted based on historical communication and signal strengths between sender and receiving nodes, this results in conserving of power since transmit power is less than maximum. DPCPLP performed quite well in comparison to the AODV and AODVPL protocols. The proposed protocol delivers better battery life, lower energy consumption, better throughput along with higher delivery rates achieved because of lower interruption time and lesser overhead packets. This is coupled with optimum transmission power levels and pre-determined alternate routes or route repairs. Thus DPCPLP brings improvement in node \& network capacity and lifetime for supporting QoS. 
There is further scope of research in testing the proposed solution in conditions where packets rates are higher and CBR packet size smaller. This is further establish suitability of this solution in real-time-traffic scenarios. We can also test other algorithms of routing \& power control and other parameters considered. E.g. Transport layer congestion control.

\section{REFERENCES}

[1] IEEE 802.11 Working Group, Wireless LAN Medium Access Control (MAC) and Physical Layer (PHY) Specification, 1997.

[2] Eun-Sun Jung, Nitin H Vaidya, “ A power Control MAC protocol For Ad Hoc Networks”, January 2005, Volume 11, Issue 1-2, pp 55-56. https://link.springer.com/article/10.1007\%2Fs11276-004-4746-9

[3] Mahesh K Marina, Samir R Das, “Ad hoc on-demand multipath distance vector routing”, 2006, Wireless Communications and Mobile Computing, DOI: 10.1002/wcm.432, John Wiley\& Sons Ltd

[4] P. Abhilash, Srinath Perur and Sridhar Iyer, Router Handoff: “An Approach for Preemptive Route Repair in Mobile Ad Hoc Networks", Proc. of High Performance Computing, 2002.

[5] Deepthy Mathews, S. Kannan, Dr. S.Karthik, "A Performance Analysis of Energy Aware and Link Stability based Routing Protocols for MANET", International Journal of Advanced Research in Compter Engineering \& Technology, Volume 1, Issue 8, October 2012.

[6] Mangesh D. Nikose, Anni U Gupta, "A case for cross layer design: The impact of physical layer properties on routing protocol performance in MANETs", February 2011, International Journal of Engineering Science and Technology, ISSN: 0975-5462, Vol 3, No. 2.

[7] Pushpender, Dr. Sohan Garg, Ram KumarM Chiang, "A study of different layers and routing protocols in wireless mesh networks", September 2013, International Journal of of Modern Computer Science and, ISSN: 2321-2632, Volume No-1, Issue No. 4.

[8] B. Jothi Mohan and V.C. Ravichandran, 2007. Cross Layer Optimization for Congestion Control in Adhoc Networks. Information Technology Journal, 6: 283-287. DOI: 10.3923/itj.2007.283.287,

[9] Dzmitry Kliazovich and Fabrizio Granelli, "Cross-layer congestion control in adhoc wireless networks", 2006.Elsevier. https://doi.org/10.1016/j.adhoc.2005.08.001

[10] C. Perkins, E. Belding Royer, S. Das, , “Ad Hoc on Demand Distance Vector (AODV) Routing”, July 2003, doi: 10.17487/RFC3561 